\title{
Vulnerabilidade de pacientes com hiperplasia prostática tratados com dutasterida e finasterida
}

\author{
Marcello Henrique Araujo da Silva ${ }^{1}$, Jady Assis de Souza ${ }^{1}$ \\ 1. Universidade do Estado do Rio de Janeiro, Rio de Janeiro/RJ, Brasil.
}

\section{Resumo}

A hiperplasia prostática benigna é uma patologia cuja incidência vem crescendo muito nos últimos anos, em todo o Brasil. A doença está correlacionada a fatores hormonais, e o tratamento farmacológico pode gerar efeitos adversos nos pacientes. O objetivo deste estudo é avaliar fatores socioeconômicos e socioculturais que interferem na cura ou reduzem a qualidade de vida. Analisamos dados de plataformas do Governo Federal entre janeiro de 2009 a setembro de 2019, observando fatores como etnia, nível de escolaridade e situação econômica dos pacientes. Em todas as regiões do Brasil esses fatores se mostraram importantes, pois podem afetar diretamente a incidência da doença e a adesão e continuidade do tratamento.

Palavras-chave: Vulnerabilidade social. Hiperplasia prostática benigna. Dutasterida. Finasterida.

\section{Resumen}

\section{Vulnerabilidad de pacientes con hiperplasia prostática tratados con dutasterida y finasterida} La hiperplasia prostática benigna es una patología cuya incidencia ha ido creciendo mucho en los últimos años, en todo Brasil. La enfermedad se correlaciona con factores hormonales, y el tratamiento farmacológico puede generar efectos adversos en los pacientes. El objetivo de este estudio es evaluar factores socioeconómicos y socioculturales que interfieren con la curación o reducen la calidad de vida. Analizamos datos de plataformas del Gobierno Federal entre enero de 2009 y septiembre de 2019, observando factores como el origen étnico, el nivel educativo y la situación económica de los pacientes. En todas las regiones de Brasil, estos factores demostraron ser importantes, ya que pueden afectar directamente la incidencia de la enfermedad y la adherencia y continuidad del tratamiento.

Palabras clave: Vulnerabilidad social. Hiperplasia prostática. Dutasterida. Finasterida.

\begin{abstract}
Vulnerability of patients with prostatic hyperplasia treated with dutasteride and finasteride Benign prostatic hyperplasia is a pathology whose incidence has been increasing in recent years throughout Brazil. The disease is correlated with hormonal factors, and pharmacological treatment can have adverse effects on patients. This study assesses the socioeconomic and socio-cultural factors that interfere with healing or reduce quality of life. We analyzed data from Federal Government platforms between January 2009 and September 2019, looking at factors such as ethnicity, education level and economic status of patients. In all regions of Brazil, these factors proved to be important, as they can directly affect the incidence of the disease and adherence and continuity of treatment.
\end{abstract}

Keywords: Social vulnerability. Prostatic hyperplasia. Dutasteride. Finasteride. 
A vulnerabilidade social é descrita como fragilidade, desfavorecimento, desamparo ou abandono. O conceito é bastante amplo e pode englobar diversas formas de exclusão ou isolamento social, de pequenos ou grandes grupos. Geralmente, a vulnerabilidade dificulta ou impede a relação com avanços, descobertas ou benefícios proporcionados pelo desenvolvimento tecnológico. No contexto da ética em pesquisa, a vulnerabilidade social é compreendida por circunstâncias capazes de afetar a qualidade de vida de um indivíduo ou grupo no que se refere à inclusão ou exclusão na sociedade ${ }^{1-3}$.

No Brasil, entre 2000 e 2010, políticas sociais reduziram a pobreza, valorizaram o salário-mínimo e formalizaram o trabalho, dentre outras ações. No entanto, nos últimos anos, a vulnerabilidade social e a miséria vêm aumentando no país ${ }^{4-5}$. Segundo o Instituto Brasileiro de Geografia e Estatística (IBGE), aproximadamente 104 milhões de brasileiros ganham menos de $R \$ 413,00$ por mês, dentre os quais 15,3 milhões vivem com menos de R\$ 140,00 por mês, em um quadro de miséria ${ }^{6}$.

Além disso, aproximadamente $73 \%$ dos pobres no Brasil são pretos ou pardos, grupos étnicos mais vulneráveis a patologias como hipertensão arterial sistêmica, diabetes mellitus tipo 2, vírus da imunodeficiência humana, doenças endócrinas e hiperplasia prostática benigna (HPB), entre outras ${ }^{7-8}$. Muitas dessas patologias estão correlacionadas a fatores ambientais e sociais e, dentre elas, a HPB vem crescendo exponencialmente nos últimos anos.

A HPB é uma doença não maligna caracterizada pelo aumento dos tecidos epiteliais e estromais e pela redução do fluxo urinário, causando distúrbios conhecidos como "sintomas do trato urinário inferior". Evidências recentes têm mostrado os mecanismos que geram ou governam a HPB. Além disso, sabemos que o envelhecimento está correlacionado à doença, que atualmente afeta aproximadamente $50 \%$ dos homens acima de 50 anos de idade e $90 \%$ dos homens na oitava década de vida ${ }^{9-12}$.

Uma das principais linhas de tratamento farmacológico indicada pela Associação Americana de Urologia e a Associação Europeia de Urologia é a prescrição de inibidores da enzima 5-alfa-redutase (5-ARI) ${ }^{13-14}$. Os 5-ARI impedem a conversão da testosterona e 5-alfa-adrostenediona em dihidrotestosterona, que é a forma ativa da testosterona, responsável pelo desenvolvimento e progressão da HPB ${ }^{11,15,16}$. O tratamento com esses medicamentos diminui a proliferação de elementos epiteliais e estromáticos, reduzindo o volume da próstata de $20 \%$ a $30 \%$, melhorando a qualidade de vida dos pacientes após 12 meses de tratamento ${ }^{17}$.

Atualmente, no Brasil, os 5-ARI cuja comercialização é autorizada pela Agência Nacional de Vigilância Sanitária (Anvisa) são a dutasterida e a finasterida. A dutasterida apresenta maiores efeitos farmacocinéticos e farmacodinâmicos que a finasterida, pois inibe as duas isoformas da enzima 5-alfa-redutase (I e II), enquanto a finasterida só inibe a isoforma do tipo $\|{ }^{18}$.

Estudos recentes têm demostrado que o tratamento farmacológico com 5-ARI para a HPB pode ter efeitos adversos, como disfunção erétil e alterações na morfologia renal ${ }^{15,16,19-21}$. Entretanto, apesar de todos os efeitos adversos relatados na literatura, a prescrição desses medicamentos ainda é considerada a melhor opção para o tratamento da HPB.

Ainda não há publicações que discutam a vulnerabilidade de homens brasileiros ao tratamento farmacológico para a HPB. Assim, o objetivo deste estudo é fazer uma análise comparativa dos seguintes dados: número de homens acima de 50 anos, cirurgias de ressecção endoscópica de próstata realizadas, biópsias de próstata, valores gastos com esse procedimento, população não alfabetizada acima de 50 anos, preços dos medicamentos-referência para o tratamento da HPB e renda per capita por etnia em todas as regiões do Brasil.

\section{Material e método}

Analisamos o perfil social segundo parâmetros da teoria principialista e a concepção de vulnerabilidade social. Além disso, comparamos a vulnerabilidade social com o possível uso de 5-ARI como abordagem terapêutica para o tratamento da HPB. Foram coletados dados dos últimos 10 anos nas seguintes plataformas de dados do Governo Federal: Departamento de Informática do Sistema Único de Saúde (Datasus), Informações de Saúde (Tabnet), Sistema de Gerenciamento de Tabela de Procedimentos, Medicamentos, Órteses, Próteses e Materiais Específicos do SUS (Sigtap) e IBGE.

Os seguintes dados foram comparados: número de homens acima de 50 anos, cirurgias de ressecção endoscópica de próstata realizadas, biópsias de próstata, valores gastos com esse procedimento, população não alfabetizada acima de 50 anos, preços dos medicamentos-referência para o tratamento da HPB e renda per capita por etnia em todas as regiões do 
Brasil. O levantamento considerou um período de 10 anos (2009-2019), e os dados foram separados pelas cinco regiões do país (Norte, Nordeste, Sul, Sudeste e Centro-Oeste). A análise e a interpretação ocorreram entre outubro de 2019 e janeiro de 2020.

Além disso, buscamos dados em fontes secundárias: PubMed, Periódicos Capes e Scientific Electronic Library Online (SciELO). Os descritores utilizados foram: "ética baseada em princípios", "vulnerabilidade social", "bioética", "hiperplasia prostática benigna", "dutasterida", "finasterida" e suas respectivas traduções na língua inglesa. Os artigos selecionados foram incluídos na pesquisa de acordo com a pertinência em relação ao assunto estudado.

\section{Discussão}

Segundo o último censo do IBGE, o Brasil tem aproximadamente 17.893.451 milhões de homens com mais de 50 anos de idade. A região do país com maior concentração desse grupo populacional é o Sudeste, com aproximadamente 8.205.826 milhões de homens nessa faixa etária, e a menor concentração está na região Norte ${ }^{22,23}$ (Tabela 1).

Estudos recentes descrevem que $50 \%$ dos homens acima de 50 anos de idade possivelmente desenvolverão HPB ${ }^{10,11}$. A biópsia de próstata é uma das ferramentas de prevenção e auxílio no diagnóstico de HPB e câncer de próstata, contudo, o número de procedimentos desse tipo realizados no Brasil ainda é inferior a quantidade de possíveis pacientes com HPB. De acordo com dados obtidos nas plataformas governamentais, foram realizadas aproximadamente 435.154 biópsias de próstata de janeiro de 2009 a setembro de 2019. Nos últimos 10 anos, o Sistema Único de Saúde (SUS) deveria ter feito aproximadamente 8.919.725 milhões de biópsias de próstata, número ideal para prevenção tanto da HPB como do câncer de próstata de acordo com a demanda apresentada ${ }^{23}$ (Tabela 1).

Após o diagnóstico de HPB, os pacientes podem passar por dois tipos de intervenção: cirúrgica (ressecção endoscópica de próstata) ou farmacológica. No SUS, o valor referente ao serviço hospitalar para ressecção endoscópica de próstata é de aproximadamente $R \$ 266,14$, e o valor gasto com profissionais é de $R \$ 328,54$, totalizando um gasto de cerca de $\mathrm{R} \$ 594,68$ por paciente. Anualmente, o Ministério da Saúde investe em torno de 7 bilhões de reais com cirurgias desse tipo, no entanto os valores não são reajustados desde 2009. O mesmo ocorre com o número de cirurgias aprovadas pelo SUS, que aparentemente estabeleceu um teto de aproximadamente 12 mil ressecções endoscópicas de próstata por ano, distribuídas ao longo do território nacional de forma desproporcional ${ }^{23}$. Dados de 2018 demonstram que, das 12.119 cirurgias aprovadas, 615 foram realizadas no Norte, 2.739 no Nordeste, 6.330 no Sudeste, 1.843 no Sul, e 592 no CentroOeste. Assim, os dados demonstram grande concentração de procedimentos na região Sudeste ${ }^{23}$.

Devido à quantidade insuficiente de biópsias de próstata, que leva a diagnósticos tardios, muitas vezes a patologia progride até que apareçam sintomas do trato urinário inferior. Nesses casos, o corpo clínico, considerando fatores como a idade do paciente, pode optar pelo tratamento farmacológico, visando maior segurança.

O preço dos 5-ARI varia nas cinco regiões do Brasil: a dutasterida custa entre $R \$ 90,22$ e $R \$ 385,56$, e a finasterida entre $R \$ 166,00$ a $R \$ 280,39^{24}$. Ao compararmos o custo dos medicamentos com a renda por etnia no Brasil, segundo dados do IBGE, observamos que entre os negros o salário médio varia de $R \$ 368,78$ a $R \$ 680,06$ por mês, entre brancos de $R \$ 679,31$ a $R \$ 1.267,25$ por mês, entre amarelos de $R \$ 461,22$ a $R \$ 1.450,82$ por mês, e entre pardos de $\mathrm{R} \$ 414,98$ a $\$ \$ 696,85$ por mês ${ }^{22,23}$ (Tabela 1).

Fica claro que a disparidade de renda entre brancos e negros pode inviabilizar o início, a aderência e a conclusão do tratamento. Embora estejam em maior número na região Sudeste, onde, teoricamente, há maior aporte tecnológico e hospitalar, indivíduos negros contam com os menores salários entre as diferentes etnias. O problema é ainda mais grave quando se considera que homens negros têm maior predisposição genética para HPB, apresentando a maior incidência da doença entre toda a população - o que mostra a vulnerabilidade a que os brasileiros estão submetidos.

Há ainda fatores socioculturais que podem influenciar direta ou indiretamente o tratamento com os 5-ARI, como o analfabetismo e o desemprego. Segundo o IBGE, atualmente o Brasil tem 11,3 milhões de analfabetos, número que corresponde a aproximadamente $6,8 \%$ da população ${ }^{25}$ (Tabela 1 ). Dados publicados em 2019 sobre o desemprego apontavam $12,3 \%$ de taxa de desocupação, o equivalente a cerca de 13 milhões de brasileiros ${ }^{26}$. 
Tabela 1. Dados analisados das plataformas do Governo Federal

\begin{tabular}{|c|c|c|c|c|c|}
\hline & Região Norte & Região Nordeste & Região Sudeste & Região Sul & Região Centro-Oeste \\
\hline $\begin{array}{l}\text { População de homens } \\
\text { (50 anos em diante) }\end{array}$ & 1.092 .926 & 4.481 .275 & 8.205 .826 & 2.907 .457 & 1.205 .967 \\
\hline Biópsia de próstataa $^{a}$ & 18.216 & 59.364 & 231.832 & 96.402 & 29.340 \\
\hline $\begin{array}{l}\text { Ressecção endoscópica } \\
\text { de próstata }^{a}\end{array}$ & 4.963 & 29.280 & 68.832 & 20.794 & 8.060 \\
\hline $\begin{array}{l}\text { População não alfabetizada } \\
\text { (50 anos em diante) }{ }^{*}\end{array}$ & 406.369 & 2.508 .591 & 1.102.219 & 371.071 & 279.550 \\
\hline Produto interno bruto (PIB) ${ }^{*}$ & 13041,58 & 9848,97 & 27141,92 & 22647,46 & 25253,47 \\
\hline $\begin{array}{l}\text { Renda per capita domiciliar } \\
\text { dos negros (reais)* }\end{array}$ & 421,12 & 368,78 & 579,72 & 580,32 & 680,06 \\
\hline $\begin{array}{l}\text { Renda per capita domiciliar } \\
\text { dos brancos (reais)* }\end{array}$ & 768,40 & 679,31 & $1.221,49$ & $1.013,03$ & $1.267,25$ \\
\hline $\begin{array}{l}\text { Renda per capita domiciliar } \\
\text { dos pardos (reais)* }\end{array}$ & 414,98 & 366,02 & 581,64 & 556,43 & 696,85 \\
\hline $\begin{array}{l}\text { Renda per capita domiciliar } \\
\text { dos amarelos (reais)* }\end{array}$ & 587,25 & 461,22 & 1450,82 & 1292,74 & 1103,77 \\
\hline
\end{tabular}

"Dados do último censo do IBGE, de 2010; a dados do período 2009-2019

O custo dos medicamentos é incompatível com a média salarial da maior parte da população brasileira. Além disso, pacientes que realizam tratamento com os 5-ARI estão propícios a patologias secundárias: redução da libido, infertilidade, disfunção erétil e insuficiência renal. Sobre esse ponto, vale ressaltar que não fornecer ao paciente orientações sobre o tratamento farmacológico e seus possíveis efeitos adversos é um fator de risco que pode afetar diretamente a qualidade de vida do indivíduo.

Em 2014, a indústria farmacêutica alcançou no Brasil um lucro recorde de US $\$ 29,4$ bilhões (cerca de $R \$ 123,2$ bilhões), e a expectativa é que em 2020 esse faturamento chegue a US $\$ 47,9$ bilhões por ano (cerca de $\mathrm{R} \$ 200,7$ bilhões). O Brasil está entre os seis maiores mercados farmacêuticos do mundo, e o crescimento é constante e exponencial ${ }^{27-29}$. Os dados revelam o grande poder comercial das farmacêuticas no país, que se estende à pesquisa científica, já que essa indústria patrocina estudos acadêmicos e tem grande influência sobre pesquisadores, médicos e formadores de opinião. Assim, tudo que é divulgado pelo mercado farmacêutico rapidamente passa a ser reivindicado por pacientes em consultórios e hospitais, levando, inclusive, à judicialização da saúde ${ }^{1}$.
Com diversos efeitos adversos relatados na literatura, alguns protocolos de tratamento farmacológico começaram a ser desenvolvidos. Um deles associa os 5-ARI com inibidores da enzima fosfodiesterase- $5^{30,31}$. Essa combinação provavelmente evita alterações no corpo cavernoso provocadas pelo uso isolado de 5-ARI. Entretanto, estudos indicam que, após o tratamento, há uma redução drástica da síntese de oxido nítrico, fundamental para o relaxamento do corpo cavernoso, gerando quadros de disfunção erétil ${ }^{21}$. Além desses dados, sabe-se que a associação de 5-ARI com inibidores da enzima fosfodiesterase- 5 causa mais alterações no pênis do que o tratamento isolado ${ }^{16}$.

Em 2010, a Anvisa autorizou a comercialização de outro medicamento, que combina 5-ARI com antagonistas alfa-1-adrenérgicos ${ }^{32}$. Esse novo protocolo teoricamente melhoraria os sintomas do trato urinário inferior, reduziria o volume da próstata e evitaria a disfunção erétil nos pacientes ${ }^{30,33-35}$. Contudo, o tratamento com antagonistas alfa-1-adrenérgicos tem efeitos adversos como depressão, ejaculação retrógrada e hipotensão arterial, entre outros ${ }^{36,37}$.

Muitos estudos já relataram alterações provocadas no sistema urogenital pelos 5-ARI, mas pouco tem se falado sobre outros efeitos adversos, 
como alterações na morfologia renal provocadas pelo tratamento farmacológico de redução do fator de crescimento endotelial nos glomérulos, aumento da fibrose na medula renal e cápsula de Bowman, além de uma perda considerável de néfrons ${ }^{19,20,38,39}$. Além disso, não se deve perder de vista que o tratamento pode afetar a qualidade de vida do paciente a médio e longo prazo.

Apesar dos efeitos colaterais, o tratamento com os 5-ARI ainda é o mais recomendado, e não deve ser abandonado pelos pacientes até que seja desenvolvido um novo fármaco com menos efeitos adversos. Nota-se, porém, dificuldade de acesso ao tratamento no país, dada a vulnerabilidade dos homens brasileiros, relativa a fatores sociais, econômicos (custo do tratamento) e éticos. É importante ressaltar, entretanto, que a Constituição Federal de 1988 define a saúde como direito de todos e dever do Estado, que é obrigado a oferecer um serviço de saúde gratuito e de qualidade para todo cidadão brasileiro. Mas, na prática, nos últimos quatro governos federais, os investimentos no SUS para diagnóstico e tratamento da HPB foram insuficientes ${ }^{40}$. A vulnerabilidade social dos homens brasileiros durante ou após o tratamento com 5-ARI também pode influenciar no aparecimento de patologias secundárias.

\section{Considerações finais}

Alguns estudos vêm demonstrando que o tratamento com inibidores da enzima 5-alfa-redutase pode causar alterações morfofisiológicas. $O$ tratamento, no entanto, continua sendo o mais recomendado para pacientes com HPB. No Brasil, os recursos financeiros do SUS para diagnosticar e tratar essa doença não são reajustados há mais de 10 anos. 0 orçamento tem se mostrado insuficiente ante a grande demanda, tanto para intervenção cirúrgica como para tratamento farmacológico. Nesse cenário adverso, assiste-se ao crescente desenvolvimento de patologias secundárias entre indivíduos em situação de vulnerabilidade.

\section{Referências}

1. Alves EF, Silva MHA, Oliveira FA, Silva TT. Vulnerabilidade social diante da fosfoetanolamina a partir da teoria principialista. Rev. bioét. (Impr.). [Internet]. 2019 [acesso 6 maio 2021];27(1):173-8. DOI: 10.1590/ 1983-80422019271300

2. Cutter SL. A ciência da vulnerabilidade: modelos, métodos e indicadores. Rev Crít Ciênc Soc [Internet]. 2011 [acesso 6 maio 2021];93:59-69. DOI: 10.4000/rccs.165

3. Carmo ME, Guizardi FL. O conceito de vulnerabilidade e seus sentidos para as políticas públicas de saúde e assistência social. Cad Saúde Pública [Internet]. 2018 [acesso 6 maio 2021];34(3):1-14. DOI: 10.1590/ 0102-311x00101417

4. Costa MA, Santos MPG, Marguti B, Pirani N, Pinto CVS, Curi RLC et al. Vulnerabilidade social no Brasil: conceitos, métodos e primeiros resultados para municípios e regiões metropolitanas brasileiras [Internet]. Brasília: Instituto de Pesquisa Econômica Aplicada; 2018 [acesso 6 maio 2021]. (Texto para Discussão; 2.364) Disponível: https://bit.ly/3uSLopk

5. Verdélio A. Vulnerabilidade social no Brasil aumenta entre 2014 e 2015. Agência Brasil [Internet]. Brasília; 23 ago 2017 [acesso 6 maio 2021]. Disponível: https://bit.ly/3uQlId5

6. Nery C. Extrema pobreza atinge 13,5 milhões de pessoas e chega ao maior nível em 7 anos. Agência de Notícias IBGE [Internet]. Estatísticas Sociais; 7 nov 2019 [acesso 6 maio 2021]. Disponível: https://bit.ly/33Nktj2

7. Negros têm maior incidência de problemas de saúde evitáveis no Brasil, alerta ONU. Canal Saúde [Internet]. Rio de Janeiro; 1 fev 2018 [acesso 6 maio 2021]. Disponível: https://bit.ly/3bwymGB

8. Ministério da Saúde. Manual de Doenças mais importantes, por razões étnicas, na população brasileira afro-descendente [Internet]. Brasília: Ministério da Saúde; 2001 [acesso 6 maio 2021]. Disponível: https://bit.ly/3uQjWZz

9. Egan KB. The epidemiology of benign prostatic hyperplasia associated with lower urinary tract symptoms: prevalence and incident rates. Urol Clin North Am [Internet]. 2016 [acesso 6 maio 2021];43(3):289-97. DOI: 10.1016/j.ucl.2016.04.001

10. Erdemir F, Harbin A, Hellstrom WJG. 5-alpha reductase inhibitors and erectile dysfunction: the connection. J Sex Med [Internet]. 2008 [acesso 6 maio 2021];5(12):2917-24. DOI: 10.1111/j.1743-6109.2008.01001.x 
11. Da Silva MHA, De Souza DB. Current evidence for the involvement of sex steroid receptors and sex hormones in benign prostatic hyperplasia. Res Rep Urol [Internet]. 2019 [acesso 6 maio 2021];11:1-8. DOI: 10.2147/RRU.S155609

12. Alcaraz A, Carballido-Rodriguez J, Unda-Urzaiz M, Medina-López R, Ruiz-Cerdá JL, Rodríguez-Rubio F et al. Quality of life in patients with lower urinary tract symptoms associated with BPH: change over time in real-life practice according to treatment: the QUALIPROST study. Int Urol Nephrol [Internet]. 2016 [acesso 6 maio 2021];48:645-56. DOI: 10.1007/s11255-015-1206-7

13. Madersbacher S, Alivizatos G, Nordling J, Sanz CR, Emberton M, de la Rosette JJ. EAU 2004 guidelines on assessment, therapy and follow-up of men with lower urinary tract symptoms suggestive of benign prostatic obstruction (BPH guidelines). Eur Urol [Internet]. 2004 [acesso 6 maio 2021];46(5):547-54. DOI: 10.1016/j.eururo.2004.07.016

14. McVary KT, Roehrborn CG, Avins AL, Barry MJ, Bruskewitz RC, Donnell RF et al. Update on AUA guideline on the management of benign prostatic hyperplasia. J Urol [Internet]. 2011 [acesso 6 maio 2021];185(5):1793-803. DOI: 10.1016/j.juro.2011.01.074

15. Da Silva MHA, Costa WS, Sampaio FJB, De Souza DB. The corpus cavernosum after treatment with dutasteride or finasteride: a histomorphometric study in a benign prostatic hyperplasia rodent model. Asian J Androl [Internet]. 2018 [acesso 6 maio 2021];20(5):505-10. DOI: 10.4103/aja.aja_28_18

16. Da Silva MHA, Medeiros JL Jr, Costa WS, Sampaio FJB, De Souza DB. Effects of the dutasteride and sildenafil association in the penis of a benign prostatic hyperplasia animal model. Aging Male [Internet]. 2019 [acesso 6 maio 2021];23(5). DOI: 10.1080/13685538.2019.1653839

17. Nickel JC, Gilling P, Tammela TL, Morrill B, Wilson TH, Rittmaster RS. Comparison of dutasteride and finasteride for treating benign prostatic hyperplasia: the Enlarged Prostate International Comparator Study (EPICS). BJU Int [Internet]. 2011 [acesso 6 maio 2021];108(3):388-94. DOI: 10.1111/j.1464-410X.2011.10195.x

18. Alcántara-Montero A, Brenes-Bermúdez FJ. Finasteride or dutasteride for the pharmacological treatment for male lower urinary tract symptoms due to benign prostatic hyperplasia? Actas Urol Esp [Internet]. 2016 [acesso 6 maio 2021];40(4):268-9. DOI: 10.1016/j.acuro.2015.12.006

19. Da Silva M, Estrada J, Sampaio F, De Souza D. The finasteride can promote kidney changes? A study developed in a model of benign prostatic hyperplasia. Eur Urol Suppl [Internet]. 2018 [acesso 6 maio 2021];17(2). DOI:10.1016/S1569-9056(18)31419-2

20. Da Silva M, Sampaio F, De Souza D. Dutasteride may change renal morphology? Eur Urol Suppl [Internet]. 2019 [acesso 6 maio 2021];18(1). DOI: 10.1016/S1569-9056(19)30181-2

21. Pinsky MR, Gur S, Tracey AJ, Harbin A, Hellstrom WJ. The effects of chronic 5-alpha-reductase inhibitor (dutasteride) treatment on rat erectile function. J Sex Med [Internet]. 2011 [acesso 6 maio 2021];8(11):3066-74. DOI: 10.1111/j.1743-6109.2011.02425.x

22. Instituto Brasileiro de Geografia e Estatística. Censo 2010 [Internet]. 2019 [acesso 6 maio 2021]. Disponível: https://bit.ly/2RlaLBK

23. Ministério da Saúde. Sistema de Gerenciamento da Tabela de Procedimentos, Medicamento e OPM do SUS [Internet]. 2019 [acesso 6 maio 2021]. Disponível: https://bit.ly/3tOaLHt

24. Avodart. Consulta Remédios [Internet]. 2019 [acesso 6 maio 2021]. Disponível: https://bit.ly/2SSOPjE

25. Ferreira P. Brasil ainda tem 11,3 milhões de analfabetos. O Globo [Internet]. Rio de Janeiro; 21 jun 2019 [acesso 6 maio 2021]. Disponível: https://glo.bo/3uQ4blm

26. PNAD Contínua: taxa de desocupação é de $12,3 \%$ e taxa de subutilização é $25,0 \%$ no trimestre encerrado em maio de 2019. Agência de Notícias IBGE [Internet]. Estatísticas Sociais; 28 jun 2019 [acesso 6 maio 2021]. Disponível: https://bit.ly/3bwx3aF

27. Leonardi E. Fatos da indústria farmacêutica nos últimos três meses. ICTQ [Internet]. Anápolis; 2018 [acesso 6 maio 2021]. Disponível: https://bit.ly/3tRZK82

28. Krugman P. A indústria farmacêutica ainda é quem manda. Exame [Internet]. São Paulo; 23 maio 2018 [acesso 6 maio 2021]. Disponível: https://bit.ly/33QFJ7e

29. Confederação Nacional dos Trabalhadores Liberais Universitários Regulamentados. Como age a indústria farmacêutica para garantir altos lucros. CNTU [Internet]. Brasília; 23 ago 2016 [acesso 6 maio 2021]. Disponível: https://bit.ly/3uQjPx7 
30. Roehrborn CG, Casabe A, Glina S, Sorsaburu S, Henneges C, Viktrup L. Treatment satisfaction and clinically meaningful symptom improvement in men with lower urinary tract symptoms and prostatic enlargement secondary to benign prostatic hyperplasia: secondary results from a 6-month, randomized, double-blind study comparing finasteride plus tadalafil with finasteride plus placebo. Int J Urol [Internet]. 2015 [acesso 6 maio 2021];22(6):582-7. DOI: 10.1111/iju.12741

31. Scaglione F, Donde S, Hassan TA, Jannini EA. Phosphodiesterase type 5 inhibitors for the treatment of erectile dysfunction: pharmacology and clinical impact of the sildenafil citrate orodispersible tablet formulation. Clin Ther [Internet]. 2017 [acesso 6 maio 2021];39(2):370-7. DOI: 10.1016/j.clinthera.2017.01.001

32. Agência Nacional de Vigilância Sanitária. Registro Anvisa $n^{\circ} 101070287$ - Combodart [Internet]. 2020 [acesso 6 maio 2021]. Disponível: https://bit.ly/3ydbeGO

33. Roehrborn CG, Manyak MJ, Palacios-Moreno JM, Wilson TH, Roos EPM, Santos JC et al. A prospective randomised placebo-controlled study of the impact of dutasteride/tamsulosin combination therapy on sexual function domains in sexually active men with lower urinary tract symptoms (LUTS) secondary to benign prostatic hyperplasia (BPH). BJU Int [Internet]. 2018 [acesso 6 maio 2021];121(4):647-58. DOI: $10.1111 /$ bju.14057

34. Roehrborn CG, Oyarzabal Perez I, Roos EPM, Calomfirescu N, Brotherton B, Wang F et al. Efficacy and safety of a fixed-dose combination of dutasteride and tamsulosin treatment (Duodart $\left(R^{\oplus}\right)$ ) compared with watchful waiting with initiation of tamsulosin therapy if symptoms do not improve, both provided with lifestyle advice, in the management of treatment-naive men with moderately symptomatic benign prostatic hyperplasia: 2-year CONDUCT study results. BJU Int [Internet]. 2015 [acesso 6 maio 2021];116(3):450-9. DOI: $10.1111 /$ bju.13033

35. Wang D, Zha X, Nagase K, Akino H, Muramatsu I, Ito H, Yokoyama O. Effects of the $5 a$-reductase inhibitor dutasteride on rat prostate alpha1A-adrenergic receptor and its mediated contractility. Urology [Internet]. 2015 [acesso 6 maio 2021];85(3):704 e9-14. DOI: 10.1016/j.urology.2014.12.002

36. Leibacher NW, Silva MHA. The Adverse effects of tamsulosin in men with benign prostatic hyperplasia, a review of the literature. Am J Life Sci [Internet]. 2019 [acesso 6 maio 2021];7(4):68-74. DOI: 10.11648/ j.ajls.20190704.11

37. Frau R, Abbiati F, Bini V, Casti A, Caruso D, Devoto P, Bortolato M. Targeting neurosteroid synthesis as a therapy for schizophrenia-related alterations induced by early psychosocial stress. Schizophr Res [Internet]. 2015 [acesso 6 maio 2021];168(3):640-8. DOI: 10.1016/j.schres.2015.04.044

38. Baig MS, Kolasa-Wolosiuk A, Pilutin A, Safranow K, Baranowska-Bosiacka I, Kabat-Koperska J et al. Finasterideinduced inhibition of 5a-reductase type 2 could lead to kidney damage-animal, experimental study. Int J Environ Res Public Health [Internet]. 2019 [acesso 6 maio 2021]16:16(10). DOI: 10.3390/ijerph16101726

39. Tian HL, Zhao CX, Wu HY, Xu ZX, Wei LS, Zhao RT, Jin DL. Finasteride reduces microvessel density and expression of vascular endothelial growth factor in renal tissue of diabetic rats. Am J Med Sci [Internet]. 2015 [acesso 6 maio 2021];349(6):516-20. DOI: 10.1097/MAJ.0000000000000451

40. Brasil. Constituição da República Federativa do Brasil [Internet]. Brasília: Senado Federal; 2019 [acesso 6 maio 2021]. Disponível: https://bit.ly/2YjZyTO

Marcello Henrique Araujo da Silva - Doutorando - marcellohas@yahoo.com.br

(i) 0000-0002-2722-9759

Jady Assis de Souza - Graduanda - jadyasouza@hotmail.com

(D) 0000-0003-2918-9209

Correspondência

Marcello Henrique Araujo da Silva - Universidade do Estado do Rio de Janeiro

Unidade de Pesquisa Urogenital. Av. 28 de Setembro, 87, Fundos, Vila Isabel CEP 20551-030.

Rio de Janeiro/RJ, Brasil.

Participação dos autores

Os autores contribuíram igualmente para o estudo.

Recebido: 19.6 .2020

Revisado: 28.4.2021

Aprovado: 7.5 .2021 